\title{
Kayaking and Wagging of Rods in Shear Flow
}

\author{
Yu-Guo Tao, W. K. den Otter, and W. J. Briels* \\ Computational Biophysics, Faculty of Science and Technology, University of Twente, \\ P.O. Box 217, 7500 AE Enschede, The Netherlands \\ (Received 14 July 2005; published 2 December 2005)
}

\begin{abstract}
For the first time, we have simulated the periodic collective orientational motions performed by rigid liquid-crystalline polymers with large aspect ratio in the nematic state in shear flow. In order to be able to do so, we developed a new, event-driven Brownian dynamics technique. We present the results of simulations of rods with aspect ratios $L / d$ ranging from 20 to 60 at volume fractions $\varphi$ given by $L \varphi / d=$ 3.5 and 4.5. By studying the path of the director, i.e., the average direction of the rods, we observe kayaking, wagging, flow aligning, and log-rolling type of orbits, depending on the parameters of the simulation and the initial orientation. We find that the tumbling periods depend on $L \varphi / d$ and the shear rate but not on the type of motion. Our simulation results qualitatively confirm theoretical predictions and are in good agreement with the experimental measurements of tumbling times of fd viruses.
\end{abstract}

PACS numbers: 61.30.Cz, 61.30.Vx, 83.10.Mj

Single elongated rigid particles in a shear flow described by $\mathbf{V}(\mathbf{r})=\dot{\gamma} y \hat{\mathbf{e}}_{\mathbf{x}}$, with $\mathbf{r}=x \hat{\mathbf{e}}_{x}+y \hat{\mathbf{e}}_{y}+z \hat{\mathbf{e}}_{z}$, trace out Jeffery orbits named after the scientist who first described this motion [1]. For example, a particle initially aligned along the flow axis $\hat{\mathbf{e}}_{\mathbf{x}}$ will start to rotate in the shear plane, i.e., the $x y$ plane, with a period depending on both the aspect ratio of the particle and the shear rate. Particles whose long axes initially make nonzero angles with the shear plane perform motions akin to that of the paddles of somebody kayaking. It is to be expected that in a dense system of elongated particles in shear flow the particles will strongly interact and will not be able to perform Jeffery motion independently of each other. If there is any tumbling at all, this has to be performed by the particles collectively. It is this rather spectacular motion that we have investigated by means of Brownian dynamics simulation and that we address in this Letter.

The dynamics of dense systems of elongated particles in shear flow was first investigated from a theoretical point of view independently by Hess [2] and Doi [3]. The central object of their investigation was the director, which is the unit vector $\hat{\mathbf{n}}$ pointing along the average direction of the long axes of all particles. A particle with long axis $\hat{\mathbf{u}}$ running through a Jeffery path $\hat{\mathbf{u}}_{J}(t)$ may be characterized by a time dependent probability density $P(\hat{\mathbf{u}}, t)=\delta(\hat{\mathbf{u}}-$ $\left.\hat{\mathbf{u}}_{J}(t)\right)$ where $\delta$ is a Dirac delta function on the twodimensional unit sphere. The corresponding evolution equation may serve as starting point to derive the equation governing the evolution of the probability density $P(\hat{\mathbf{n}}, t)$ of the director of a dense system of elongated particles. To this end we must add to the original evolution equation terms which account for perturbations of the Jeffery paths caused by the interactions between particles and by the random kicks exerted by the solvent molecules on these particles. Doing so, we arrive at the Smoluchowski equation first derived by Hess and Doi.

The initial motivation for deriving the Doi-Hess equation was to obtain expressions relating the aspect ratio of the particles to the various coefficients occurring in the phenomenological theory by Ericksen [4] and Leslie [5]. The latter theory, in combination with results obtained by means of the Doi-Hess equation, has been very successful in describing the rich rheology of dense rodlike particles systems. Besides describing rheology, solutions of the DoiHess equation provide detailed molecular information which is difficult to obtain experimentally. Over the last two decades the following picture has emerged: at low shear rates and sufficiently high volume fractions, all particles collectively tumble in the shear plane [6-8] or perform Jeffery type of motions [9] called kayaking. Tumbling is only observed if the director is initially in the shear plane. Recently, however, it has been claimed [10] that tumbling always is unstable with respect to kayaking. With increasing shear rates tumbling and kayaking go over into wagging [8-10] and finally into a shear aligned state [6-10]. In the wagging state the director periodically moves up and down in the shear plane in a symmetrical way about the flow direction. In the shear aligned state the director is arrested [8] in the shear plane making a fixed nonzero angle with the flow direction. There is one more stationary state, one in which the director is initially aligned along the vorticity direction, i.e., the $z$ axis, and remains there; this state is called the log-rolling state. For a more complete description of all possible motions and their dependence on the initial state, we refer to Hess and Kröger [11,12] and references given there. Recently, a thorough analysis of the predictions of the DoiHess equation, avoiding any additional approximation not implicit in the use of the Doi-Hess equation, has been published by Forest et al. [13,14].

The Doi-Hess equation from which the above picture has emerged is based on a number of approximations which were itemized by Dhont and Briels [15]. Recently we have shown by means of Brownian dynamics simulations that, at low volume fractions of suspended rods, these have severe consequences for the predictions made by the 
theory [16]. In particular, the theory neglects dynamic correlations between neighboring rods and it may therefore be questioned if it is capable of correctly describing collective motions in dense systems. Moreover, although periodic responses have been observed in rheological experiments [17-19], no simple measurements exist discriminating between the various kinds of periodic motions. In this situation, computer experiments simulating the full $\mathrm{N}$-particle Fokker-Planck equation will be of great value. Only very few attempts in this direction have been undertaken. Brownian dynamics simulations by Doi et al. $[20,21]$ are restricted to elongated particles in the semidilute isotropic state. The simulations of Mori et al. [22,23] do address the dense state, but are restricted to particles with aspect ratio equal to 3 . In the first case, overlap between particles was prohibited by very steep, short range lubrication forces, in the second case by a Gay-Berne potential. Such forces become increasingly difficult to handle with increasing aspect ratio and volume fraction. We therefore set out to develop a new, event-driven Brownian dynamics algorithm which efficiently handles dense systems of Brownian hard rods, and to perform simulations of such systems in shear flow. The novelty of the code is in the fact that its time evolution is controlled by collisions, like in time of flight molecular dynamics simulations, and not by a potential preventing overlaps.

The simulation was done by means of an event-driven Brownian dynamics code neglecting hydrodynamic interaction between rods. During each time step $\Delta t$ the rods were propagated from position $\mathbf{r}(t)$ to

$$
\mathbf{r}(t+\Delta t)=\mathbf{r}(t)+\mathbf{\Gamma} \cdot \mathbf{r}(t) \Delta t+\delta \mathbf{r}(t)
$$

while their orientations were changed from $\hat{\mathbf{u}}(t)$ to

$$
\hat{\mathbf{u}}(t+\Delta t)=\hat{\mathbf{u}}(t)+[\hat{\mathbf{I}}-\hat{\mathbf{u}}(t) \hat{\mathbf{u}}(t)] \cdot \boldsymbol{\Gamma} \cdot \hat{\mathbf{u}}(t) \Delta t+\delta \hat{\mathbf{u}}(t),
$$

where $\boldsymbol{\Gamma}$ is a matrix with $\Gamma_{x y}=\dot{\gamma}$ and all remaining elements equal to zero and $\hat{\mathbf{I}}$ is the unit matrix. The random displacements and reorientations were drawn according to

$$
\begin{gathered}
\langle\delta \mathbf{r}(t) \delta \mathbf{r}(t)\rangle=2 k_{B} T \Xi^{-1}(t) \Delta t, \\
\langle\delta \hat{\mathbf{u}}(t) \delta \hat{\mathbf{u}}(t)\rangle=2 k_{B} T \gamma_{r}^{-1}[\hat{\mathbf{I}}-\hat{\mathbf{u}}(t) \hat{\mathbf{u}}(t)] \Delta t,
\end{gathered}
$$

where

$$
\begin{gathered}
\Xi=\gamma_{\|} \hat{\mathbf{u}}(t) \hat{\mathbf{u}}(t)+\gamma_{\perp}[\hat{\mathbf{I}}-\hat{\mathbf{u}}(t) \hat{\mathbf{u}}(t)], \\
\gamma_{r}=\frac{\pi \eta L^{3}}{3 \ln (L / d)}, \quad \gamma_{\|}=\frac{2 \pi \eta L}{\ln (L / d)}, \quad \gamma_{\perp}=2 \gamma_{\|},
\end{gathered}
$$

with $\eta$ the viscosity of the solvent and $T$ the temperature. Equation (6) is sufficiently accurate for the large aspect ratios used in this Letter. After each time step all pairs were checked for possible overlaps. For each overlapping pair, the collision time $t+\lambda_{c} \Delta t$ was detected by interpolation between $t$ and $t+\Delta t$ using the already chosen random displacements and reorientations, but with $\Delta t$ replaced by $\lambda_{c} \Delta t$. Next the pair was propagated to the point of contact and the remaining part of the time step was completed in a similar way. Results of simulations at low volume fractions were in excellent agreement with those of previous simulations [16] using conventional Brownian dynamics using stiff potential interactions to prevent overlaps between the rods. The current code, however, is more efficient and can be operated at higher densities and larger aspect ratios.

In Fig. 1 we present four snapshots of a simulation box containing 1750 rods with aspect ratio $L / d=25$ at a volume fraction $\varphi$ determined by $L \varphi / d=3.5$. For all simulations discussed in this Letter the diameter $d=$ $0.0148 \mu \mathrm{m}$, so the length $L=0.37 \mu \mathrm{m}$ in this case. The solvent is always water with viscosity $\eta=1 \mathrm{mPas}$ and temperature $\mathrm{T}=300 \mathrm{~K}$. A time step $\Delta t$ was chosen equal to $0.5 \mu \mathrm{s}$.

The boxes shown in Fig. 1 were sheared with shear rate $\dot{\gamma}=125 \mathrm{~s}^{-1}$. In frame (a) the rods are on average aligned along the flow direction. Half of each rod is light gray, the other half dark gray. The coloring is chosen such that for each rod in frame (a) the dark part is to the right of the light part. As time advances the rods collectively rotate such that the dark parts point southeast in frame (b) and southwest somewhat later in frame (c). Finally in frame (d) the rods are aligned along the flow direction again, but now with their dark parts to the left of the light parts; i.e., they made a turn of $180^{\circ}$ around the $z$ axis. Since the particles are symmetric, this state is indistinguishable from the original state (a). We stress that the tumbling of the rods is not the result of a rotation of the whole box, but that it is really a rotation of the individual rods performed collectively, (a)

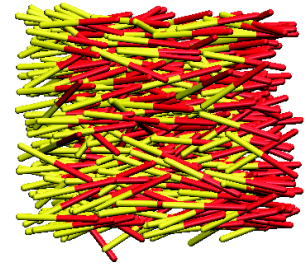

(c)

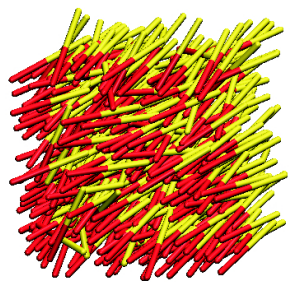

(b)

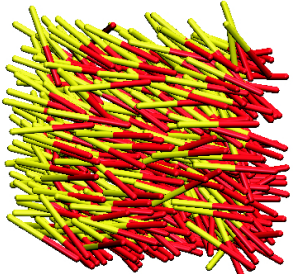

(d)

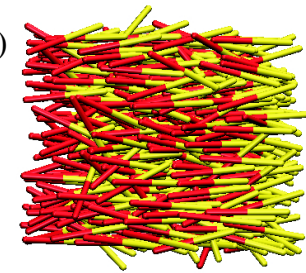

FIG. 1 (color online). Snapshots of a simulation box with rods collectively changing orientation by $180^{\circ}$. The four snapshots are taken at the times indicated as (a), (b), (c), and (d), respectively, in the upper part of Fig. 2. The box contains 1750 rods of which only 500 are shown in each frame, so the density is 3.5 times as high as it appears from the pictures. 
without appreciably changing position except along the flow direction.

The details of the motion can hardly be seen from a time series of snapshots like in Fig. 1. Therefore we plot in Fig. 2 the three components $\hat{n}_{x}, \hat{n}_{y}$, and $\hat{n}_{z}$ of the director $\hat{\mathbf{n}}$ together with the order parameter as a function of time. At each time the order tensor $\mathbf{S}=N^{-1} \sum_{i} \hat{\mathbf{u}}_{i}(t) \hat{\mathbf{u}}_{i}(t)-\frac{1}{3} \hat{\mathbf{I}}$ was calculated and diagonalized. The eigenvector with the largest eigenvalue is the director $\hat{\mathbf{n}}(t)=\hat{n}_{x}(t) \hat{\mathbf{e}}_{\mathbf{x}}+$ $\hat{n}_{\mathbf{y}}(t) \hat{\mathbf{e}}_{y}+\hat{n}_{z}(t) \hat{\mathbf{e}}_{\mathbf{z}}$ and is characteristic for the average direction of the long axes of the particles. The corresponding eigenvalue is the order parameter $P_{2}$, which is equal to zero if the rods have random orientations and equal to one in case the rods are all pointing in the same direction. The $x$ component of the director is seen to periodically change from +1 to -1 and back again. This confirms what we inferred from inspection of Fig. 1.

More interesting is the time evolution of $\hat{n}_{y}$ and $\hat{n}_{z}$. At those time spans when $\hat{n}_{x}$ is changing from +1 to -1 or the other way round, $\hat{n}_{z}$ drops from about -0.25 to about -0.75 and returns to -0.25 again. At the same time $\hat{n}_{y}$ alternatingly drops from zero to about -0.75 and returns to zero or rises from zero to +0.75 in order to return to zero again. This behavior is indicative of the motion called kayaking. Note that the motion is such that the average orientation remains to be along the flow direction for a while and that the actual flip to the reverse orientation occurs during a rather short time span. The results pre-
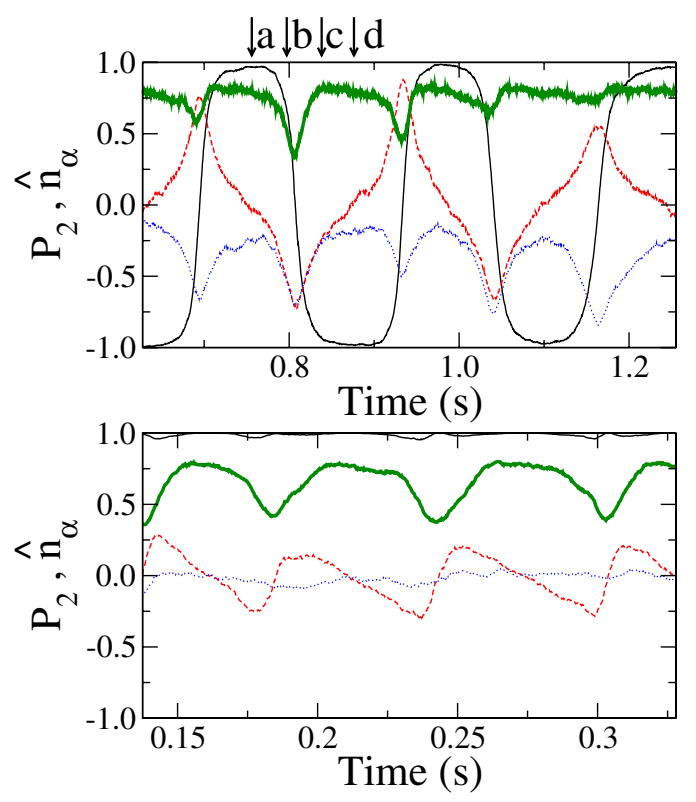

FIG. 2 (color online). Order parameter $P_{2}$ (thick solid line) and director components $\hat{n}_{x}$ (solid line), $\hat{n}_{y}$ (dashed line), and $\hat{n}_{z}$ (dotted line) as a function of time. The upper and the lower figures illustrate the kayaking and wagging motions, respectively. In this figure are also indicated the times at which the snapshots in the upper part of Fig. 1 were taken. sented in this figure and elsewhere in this Letter have been confirmed with simulation boxes with volumes twice, four and eight times as large as the present one.

We now summarize our findings on the basis of a large number of simulations with $L / d$ ranging from 20 to 60 for two values of $L \varphi / d$ and $\dot{\gamma}$ ranging from 30 to $1000 \mathrm{~s}^{-1}$. A detailed description will be published elsewhere. At low shear rates and for all initial conditions except when initially the director is aligned along the vorticity direction, the system ends up kayaking with a period which depends on $\dot{\gamma}$ and on $L \varphi / d$. In particular, tumbling turns out to be unstable with respect to kayaking except maybe for the smallest aspect ratio. A picture of the path traced out by the director during one period is drawn in Fig. 3(a) as well as in the upper part of Fig. 2. At intermediate shear rates and for the same initial conditions, the director moves towards the shear plane in order to perform a wagging motion, as illustrated in the lower part of Fig. 2 and Fig. 3(b). The small value of $\hat{n}_{z}$ indicates an in-plane motion and the alternation of $\hat{n}_{y}$ around zero denotes the wagging along the shear direction. There seems to be no abrupt change of the period at the kayaking-to-wagging transition. At even higher shear rates the director gets arrested in the shear plane making a nonzero angle with the $x$ axis. In case the director initially starts along the vorticity direction, it ends up performing small rotations around its initial direction. At high enough shear rates, this state becomes unstable and the director moves towards the shear plane where it gets

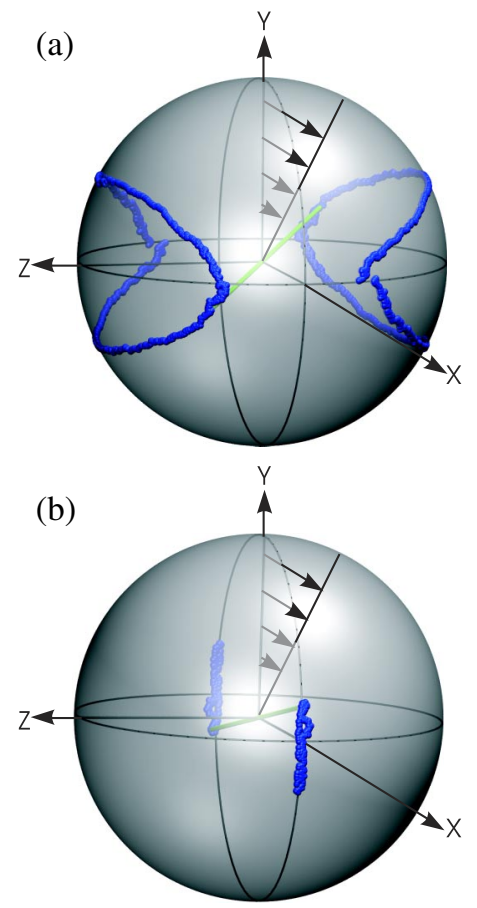

FIG. 3 (color online). The path on the unit sphere traced out by the director of a kayaking (a) and wagging (b) box of rodlike particles with $L / d=25$ and $L \varphi / d=3.5$. The arrows attached to the $y$ axis represent the flow field. 


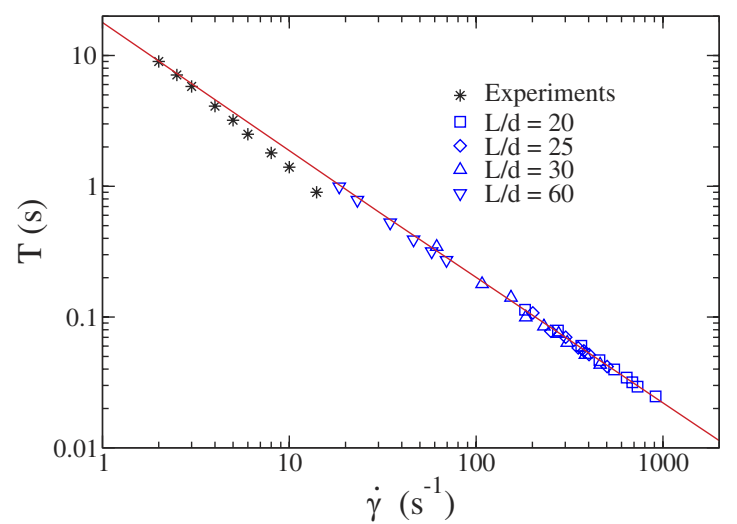

FIG. 4 (color online). Simulated and experimental tumbling times $T$ as a function of the shear rate $\dot{\gamma}$ at a volume fraction $\varphi$ determined by $L \varphi / d=4.5$. Experiments were done [24] on the fd virus for which $L / d=60$ and $d=0.0148 \mu \mathrm{m}$ like in our simulations.

arrested in the same unique state, for the given shear rate, where all other systems end. So it turns out that the results obtained so far are in qualitative agreement with the predictions of the Doi-Hess theory. Many more simulations will be needed to construct a phase diagram as has been done on the basis of the Doi-Hess equation [11-14].

As a quantitative test of our simulation method, we plot in Fig. 4 the tumbling time $T$ as a function of shear rate $\dot{\gamma}$ for all simulations with $L \varphi / d=4.5$. In the same plot we present experimental results on the fd virus [24], which has an effective diameter equal to the one that we have used and for which $L / d=60$. Using a molar weight of $1.64 \times$ $10^{7} \mathrm{~g} / M$ [25], our simulations correspond to a density of $13.6 \mathrm{mg} / \mathrm{ml}$ while the experimental density is $13 \mathrm{mg} / \mathrm{ml}$. Our results, when extrapolated to the experimental shear rates, are larger than the experimental ones by a factor of at most 1.5 . Since the fd virus is slightly flexible, its tumbling periods are expected to be smaller [26] than those of the stiff particles which were used in the simulations, hence we consider the agreement good. It will be interesting to investigate if the difference between the slopes of the experimental and the simulated curves may also be attributed to this difference in flexibility. As a second test we mention that for $L / d=60$, the transition from kayaking to wagging was found to occur between $\dot{\gamma}=27.8$ and $30.1 \mathrm{~s}^{-1}$, whereas the experimental transition occurs at $\dot{\gamma} \approx 30 \mathrm{~s}^{-1}$ when the density is $14 \mathrm{mg} / \mathrm{ml}$ [24].

From Fig. 4 we infer that the tumbling period is proportional to $1 / \dot{\gamma}$, the constant of proportionality only depending on $L \varphi / d$. Now consider two neighboring rods displaced along the $y$ axis over a distance $l$. During a period $T$ they get displaced along the flow direction over a distance $T \dot{\gamma} l$. Apparently, in order to synchronize the tumbling of all rods, a neat correlation must exist between the rotational motions and the relative displacements of the rods along the flow direction.
This work is part of the SoftLink research program of the Stichting voor Fundamenteel Onderzoek der Materie (FOM), which is financially supported by the Nederlandse Organisatie voor Wetenschappelijk Onderzoek (NWO). The authors thank Dr. J.T. Padding and Professor J. K. G. Dhont for many useful discussions. We thank Dr. M.P. Lettinga for making available his data before publication.

*Electronic address: w.j.briels@utwente.nl

[1] G. B. Jeffery, Proc. R. Soc. Lond., Ser. A 102, 161 (1922).

[2] S. Hess, Z. Naturforsch., A: Phys., Phys. Chem., Kosmophys. 31, 1034 (1976).

[3] M. Doi, J. Polym. Sci., Polym. Phys. Ed. 19, 229 (1981).

[4] J. L. Ericksen, Arch. Ration. Mech. Anal. 4, 231 (1960).

[5] F. M. Leslie, Arch. Ration. Mech. Anal. 28, 265 (1968).

[6] N. Kuzuu and M. Doi, J. Phys. Soc. Jpn. 53, 1031 (1984).

[7] G. Marrucci and P. L. Maffettone, Macromolecules 22, 4076 (1989).

[8] R. G. Larson, Macromolecules 23, 3983 (1990).

[9] R. G. Larson and H. C. Öttinger, Macromolecules 24, 6270 (1991).

[10] V. Faraoni, M. Grosso, S. Crescitelli, and P. L. Maffettone, J. Rheol. (N.Y.) 43, 829 (1999).

[11] S. Hess and M. Kröger, J. Phys. Condens. Matter 16, S3835 (2004).

[12] M. Kröger, Phys. Rep. 390, 453 (2004).

[13] M. Forest, Q. Wang, and R. H. Zhou, Rheol. Acta 43, 17 (2004).

[14] M. Forest, Q. Wang, and R. H. Zhou, Rheol. Acta 44, 80 (2004).

[15] J. K. G. Dhont and W. J. Briels, Colloids and Surfaces A 213, 131 (2003).

[16] Y.-G. Tao, W. K. den Otter, J. T. Padding, J. K. G. Dhont, and W. J. Briels, J. Chem. Phys. 122, 244903 (2005).

[17] P. Moldenaers, G. Fuller, and J. Mewis, Macromolecules 22, 960 (1989).

[18] W. R. Burghardt and G. G. Fuller, Macromolecules 24, 2546 (1991).

[19] M. Grosso, S. Crescitelli, E. Somma, J. Vermant, P. Moldenaers, and P. L. Maffettone, Phys. Rev. Lett. 90, 098304 (2003).

[20] Y. Yamane, Y. Kaneda, and M. Doi, J. Non-Newtonian Fluid Mech. 54, 405 (1994).

[21] Y. Yamane, Y. Kaneda, and M. Doi, J. Phys. Soc. Jpn. 64, 3265 (1995).

[22] N. Mori, R. Semura, and K. Nakamura, Mol. Cryst. Liq. Cryst. 367, 3233 (2001).

[23] N. Mori, H. Fujioka, R. Semura, and K. Nakamura, Rheol. Acta 42, 102 (2003).

[24] M.P. Lettinga, Z. Dogic, H. Wang, and J. Vermant, Langmuir 21, 8048 (2005).

[25] T. A. Lenstra, Z. Dogic, and J. K. G. Dhont, J. Chem. Phys. 114, 10151 (2001).

[26] F. Greco and G. Marrucci, Liq. Cryst. 22, 11 (1997). 